\title{
Active Unmatched Disturbance Cancellation and Estimation by State-Derivative Feedback for Plants Modeled as an LTI System
}

\author{
Halil Ibrahim Basturk \\ Department of Mechanical Engineering, Bogazici University, Turkey \\ halil.basturk@boun.edu.tr
}

\section{ARTICLE INFO}

Article History:

Received 27 July 2017

Accepted 09 March 2018

Available 20 July 2018

Keywords:

Adaptive Control

Estimation

Backstepping

Disturbance Cancellation

AMS Classification 2010:

93C40, 93E10, 93D21

\author{
ABSTRACT
}

We design adaptive algorithms for both cancellation and estimation of unknown periodic disturbance, by feedback of state-derivatives (i.e., without position information for mechanical systems) for the plants which are modeled as a linear time invariant system. We consider a series of unmatched unknown sinusoidal signals as the disturbance. The first step of the design consists of the parametrization of the disturbance model and the development of observer filters. The result obtained in this step allows us to use adaptive control techniques for the solution of the problem. In order to handle the unmatched condition, a backstepping technique is employed. Since the partial measurement of the virtual inputs is not available, we design a state observer and the estimates of these signals are used in the backstepping design. Finally, the stability of the equilibrium of the adaptive closed loop system with the convergence of states is proven. As a numerical example, a two-degree of freedom system is considered and the effectiveness of the algorithms are shown.

\section{Introduction}

Active disturbance cancellation arises in many applications such as automotive [1] and marine systems $[2[4]$. The internal model principle which relies on modeling the disturbance as the output of an exosystem and duplicating its effect to the input channel, is the one of the most popular technique for the solution $[5,6$.

The internal model principle differs according to the type of the considered system, uncertainties and the measured signals. In [7], the harmonic control arrays is employed for the parametrization of the sinusoidal signals. The solutions for linear systems are given in 8 11. The plants that contain nonlinear dynamics are proposed in [12 15]. Most of disturbance cancellation problems can also be formulated as an output regulation problem. In these references, it is assumed that the measurements of the state are available for feedback. Since the accelerometer is the major sensor that are used in mechanical applications, the direct usage of the acceleration measurement in the feedback draws the attention of the researchers.

In [16], acceleration measurement is used together with velocity and position to suppress the vibration. The term state-derivative feedback is widely used in the literature for the case where only the derivative of the considered states are available for measurement. That corresponds to acceleration and velocity feedback without using position information in mechanical systems. The pole placement technique and stability results for statederivative is given in 17 and [18. The adaptive cancellation algorithms by state derivative feedback for matched disturbance in a known linear plant is given in [19]. The solution for the case where the unknown disturbance is unmatched and the state of the actuator dynamics is available for the measurement is given in [20]. 
In this note, the problem where the actuator states are not measured but its derivative is available for a control design, is considered. The design by the derivative of the actuator state is given in [21]. The main difference between 21] is the number of the state of the actuator dynamics. In the present study, an arbitrary $m-$ dimensional general linear system is considered for the actuator dynamics whereas it is restricted by one, in 21]. We consider two cases; first case is state and disturbance estimation in the open loop, and the second case is disturbance cancellation in the closed loop case. Firstly, a parametrization of the unknown disturbance signal is performed. Observers are developed for the unknown disturbance and the unmeasured actuator state for open loop case. We show that perfect state and observer estimations are achieved. The results that are obtained in the open loop case, is used while designing an active cancellation algorithm for the control input. An adaptive control technique is employed for the design of observer based control and update laws. Finally, we prove the stability of the equilibrium of the closed-loop adaptive system and the convergence of the state with perfect disturbance estimation.

The outline of the note is given as follows. Section 2 is dedicated to state the problems in details. The observer designs, theorem that states the convergence and its proof are given in Section 3 . The backstepping adaptive controller design with the statement of the stability theorem is presented in Section 4. The theorem is proven in Section 5. As a numerical example, a two-degree of freedom mechanical system is considered. The simulation results of the example are discussed in Section 6. Lastly, concluding remarks are given in Section 7 .

\section{Problem statement}

We consider a general representation of a linear time invariant system which is given as follows

$$
\begin{aligned}
& \dot{x}(t)=A_{n} x(t)+B_{n}\left(b_{x}^{T} p(t)+a_{x}^{T} x+\nu(t)\right), \\
& \dot{p}(t)=A_{m} p(t)+B_{m}\left(b_{p}^{T} p(t)+a_{p}^{T} x+u(t)\right),
\end{aligned}
$$

where

$$
\begin{gathered}
A_{n}=\left[\begin{array}{cc}
0_{n-1} & I_{n-1} \\
0 & 0_{n-1}^{T}
\end{array}\right], A_{m}=\left[\begin{array}{cc}
0_{m-1} & I_{m-1} \\
0 & 0_{m-1}^{T}
\end{array}\right], \\
B_{n}=\left[\begin{array}{c}
0_{n-1} \\
1
\end{array}\right], \quad B_{m}=\left[\begin{array}{c}
0_{m-1} \\
1
\end{array}\right],
\end{gathered}
$$

$$
\begin{aligned}
& a_{x}=\left[a_{11}, \cdots, a_{1 n}\right]^{T}, \quad a_{p}=\left[a_{21}, \cdots, a_{2 n}\right]^{T}, \\
& b_{x}=\left[b_{11}, \cdots, b_{1 n}\right]^{T}, \quad b_{p}=\left[b_{21}, \cdots, b_{2 n}\right]^{T},
\end{aligned}
$$

with $0_{i}=[0, \ldots, 0]^{T} \in \mathbb{R}^{i}$, the states $x=$ $\left[x_{1}, \ldots, x_{n}\right]^{T} \in \mathbb{R}^{n}, p=\left[p_{1}, \ldots, p_{m}\right]^{T} \in \mathbb{R}^{m}$, input $u \in \mathbb{R}$ and disturbance signal $\nu \in \mathbb{R}$ given by

$$
\nu(t)=\sum_{i=1}^{q} g_{i} \sin \left(\omega_{i} t+\phi_{i}\right),
$$

where $i \neq j \Rightarrow \omega_{i} \neq \omega_{j}, \omega_{i} \in \mathbb{Q}_{+}, g_{i}, \phi_{i} \in \mathbb{R}$. In the given system, the disturbance signal and the control input are separated by $m$ integrators. This situation makes the input $u(t)$ and $\nu(t)$ unmatched. The input of system (1) is $b_{x}^{T} p(t)$ that is called virtual input. Because $b_{x}^{T} p(t)$ is not the main control input, and it has its own dynamics given by (2). The main aim is to design a control law for $u(t)$ such that the signal $b_{x}^{T} p(t)$ cancels the effect of the disturbance while maintaining the boundedness of all signals. Since state $p(t)$ and disturbance $\nu(t)$ are not measured, observers are designed to estimate these signals.

The disturbance $\nu(t)$ that is given by (7) can be also written as the output of a linear exosystem which is given by,

$$
\begin{aligned}
\dot{w}(t) & =S w(t) \\
\nu(t) & =h^{T} w(t)
\end{aligned}
$$

where $w \in \mathbb{R}^{2 q}$. The eigenvalues of $S$ depend on the unknown frequencies of disturbance signal $\nu(t)$. Since the initial condition of the exosystem, $(w(0))$ represents the uncertainty of amplitude and phase, the output vector $h^{T}$ can be considered as known. Therefore, it is possible to obtain an observable $\left(h^{T}, S\right)$ pair.

In order to guarantee the invertibility and to avoid any discontinuity in the design, it is assumed that the terms $a_{11} \neq 0$ and $b_{21} a_{11}-a_{21} b_{11} \neq 0$ (Assumption 1). We also assume that the number of the distinct frequencies, $q$ is known (Assumption 2). The given assumptions are sufficient for observer based control design and show the boundedness of signals. However, the all modes of the exosystem assumed to be excited (i.e., $w(0) \neq 0)$ to show the perfect state and disturbance estimations, as well as, the convergence of the state $x(t)$ (Assumption 3).

The main difference between the problem statement given in this note and [21] is the dimension of $p(t)$. In 21] $p(t)$ is restricted as a scalar signal. 
However, in this note there is not any restriction on the dimension of $p(t)$ and it can be an $m$ dimensional vector where $m$ is an arbitrary positive integer. Thus this note provides a solution for more general systems.

\section{State and disturbance observer design}

This section consists of two parts; the first part explains the details of parametrization and observer filters of disturbance signal, in the second part the state observer design for the virtual control input $p(t)$ is given.

\subsection{Parametrization of disturbance signal}

The disturbance representation given by (8) is not suitable for an observer design since the eigenvalues of system matrix $S$ are on the imaginary axis. The first step of observer design for disturbance is to represent it as the output of a stable LTI system. To this end, we employ the approach given in 22. Consider the Sylvester equation

$$
M S-G M=l h^{T} .
$$

where $M \in \mathbb{R}^{2 q \times 2 q}$ is the solution of the equation. As it is discussed in [23], the solution $M$ is unique and invertible if and only if $\left(h^{T}, S\right)$ is an observable pair, $(G, l)$ is a controllable pair and the set of eigenvalues of $S$ and $G$ are discrete. Therefore, by choosing $G \in \mathbb{R}^{2 q \times 2 q}$ as a Hurwitz matrix and vector $l \in \mathbb{R}^{2 q}$ such that the pair $(G, l)$ is controllable, we guarantee that $M$ is unique and invertible. We transform state of the exosystem $w(t)$ to a new state $z(t)$ as follows $z=M w$. By using 10 with the state transformation, exosystem (8)-(9) is transformed into the form

$$
\begin{aligned}
\dot{z}(t) & =G z(t)+l \nu(t), \\
\nu(t) & =\theta^{T} \dot{z}(t),
\end{aligned}
$$

where

$$
\theta^{T}=h^{T}(M S)^{-1}
$$

It is also possible to obtain the derivative of disturbance $\nu(t)$ by differentiating (12) and substituting $\ddot{z}=G \dot{z}+l \dot{\nu}$. The parametrized form of disturbance derivative is given by

$$
\dot{\nu}(t)=\beta^{T} \dot{z}(t),
$$

where

$$
\beta^{T}=\frac{1}{1-\theta^{T} l} \theta^{T} G
$$

It is necessary to show that the term $1-\theta^{T} l$, that appears in the denominator is not equal to zero. Post-multiplying $(10)$ by $(M S)^{-1}$ and substituting (13), we obtain the following equation

$$
I-l \theta^{T}=G M S^{-1} M^{-1} .
$$

Calculating the determinant of both sides of (16) and employing the Sylvester's determinant theorem 24 yield

$$
1-\theta^{T} l=\operatorname{det}\left(G M S^{-1} M^{-1}\right)
$$

Recalling that $\operatorname{det}\left(G M S^{-1} M^{-1}\right)=\operatorname{det}(G) \operatorname{det}(M)$ $\operatorname{det}\left(S^{-1}\right) \operatorname{det}\left(M^{-1}\right)$ and $\operatorname{det}\left(M^{-1}\right)=\frac{1}{(\operatorname{det} M)}$, we obtain

$$
1-\theta^{T} l=\operatorname{det}(G) \operatorname{det}\left(S^{-1}\right) .
$$

Recalling the fact that the determinant of a matrix is the multiplication of its eigenvalues and noting that $G$ and $S$ have no eigenvalues on the origin, we show that $1-\theta^{T} l \neq 0$.

The disturbance signal and its derivative are represented as the output of a known and stable LTI system whose input is itself, ans thus, unknown. It is now possible to design an observer to estimate state $z(t)$, since it is the state of exponentially stable linear time invariant system. We present the following lemma that explains the benefits of developed observer filters.

Lemma 1. The unknown disturbance $\nu(t)$ and $\dot{\nu}(t)$ are represented in the form

$$
\begin{aligned}
& \nu(t)=\theta^{T} \xi(t)+\theta^{T} \delta(t), \\
& \dot{\nu}(t)=\beta^{T} \xi(t)+\beta^{T} \delta(t),
\end{aligned}
$$

where

$$
\begin{aligned}
\xi(t)= & \eta(t)+N \dot{x}(t), \\
\dot{\eta}(t)= & G(\eta(t)+N \dot{x}(t))-N\left(A_{n} \dot{x}(t)+B_{n}\left(b_{x}^{T} \dot{p}(t)\right.\right. \\
& \left.\left.+a_{x}^{T} \dot{x}(t)\right)\right),
\end{aligned}
$$

with

$$
N=\frac{1}{B_{n}^{T} B_{n}} l B_{n}^{T},
$$

where 


$$
N B_{n}=l
$$

and the estimation error

$$
\delta(t)=\dot{z}(t)-\xi(t),
$$

obeys the equation

$$
\dot{\delta}(t)=G \delta(t)
$$

Proof. Using the fact that $N A_{n}=0$ from (3), (4) and (24), the equation (26) is obtained by differentiating (25) and using derivative of (1), (11) and (21). Substitution of (25) into (12) and (14) yields (19) and (20), respectively.

The new representation of the disturbance given in (19) and (20) provides benefits to use adaptive control method in the design. In the next section, the design for the state observer together with update law for unknown constant $\theta$ are discussed.

\subsection{Reciprocal state-space representation and observer design}

A standard state space form of a linear system is useful for a state or output feedback design. On the other hand, the reciprocal state-space (RSS) representation that relies on switching state and state-derivatives in the equation, provides advantages for state-derivative feedback design [16]. In this form, it is possible to employ similar techniques that are developed for state and output feedback problems.

Substituting (19) into (1), the RSS form of system (1) is written as

$$
\begin{aligned}
x(t)= & A_{n}^{T} \dot{x}(t)+\frac{1}{a_{11}} \bar{B}_{n}\left(\dot{x}_{n}(t)-a_{x}^{T} A_{n}^{T} \dot{x}(t)\right. \\
& \left.-b_{x}^{T} p(t)-\left(\theta^{T} \xi(t)+\theta^{T} \delta(t)\right)\right) .
\end{aligned}
$$

where $\bar{B}_{n}=\left[\begin{array}{ll}1, & 0_{n-1}\end{array}\right]^{T}$. Substituting 27) into (2), we obtain

$$
\begin{aligned}
\dot{p}(t)= & A_{m} p(t)+B_{m}\left(\bar{b}_{p}^{T} p(t)+\bar{a}_{p}^{T} A_{n}^{T} \dot{x}(t)+\frac{a_{21}}{a_{11}}\right. \\
& \left.\times \dot{x}_{n}(t)-\frac{a_{21}}{a_{11}}\left(\theta^{T} \xi(t)+\theta^{T} \delta(t)\right)+u(t)\right),
\end{aligned}
$$

where

$$
\begin{aligned}
& \bar{a}_{p}=a_{p}-\frac{a_{21}}{a_{11}} a_{x}=\left[\bar{a}_{1}, \cdots, \bar{a}_{n}\right]^{T}, \\
& \bar{b}_{p}=b_{p}-\frac{a_{21}}{a_{11}} b_{x}=\left[\bar{b}_{1}, \cdots, \bar{b}_{n}\right]^{T} .
\end{aligned}
$$

Using the fact $\bar{b}_{1}=\frac{a_{11} b_{21}-b_{11} a_{21}}{a_{11}}$, under Assumption 1 and 2, the RSS form of (28) is obtained as

$$
\begin{aligned}
p(t)= & A_{m}^{T} \dot{p}(t)+\frac{1}{\bar{b}_{1}} \bar{B}_{m}\left(\dot{p}_{m}(t)-\bar{b}_{p}^{T} A_{m}^{T} \dot{p}(t)\right. \\
& -\bar{a}_{p}^{T} A_{n}^{T} \dot{x}(t)-\frac{a_{21}}{a_{11}} \dot{x}_{n}(t) \\
& \left.+\frac{a_{21}}{a_{11}}\left(\theta^{T} \xi(t)+\theta^{T} \delta(t)\right)-u(t)\right)
\end{aligned}
$$

where $\bar{B}_{m}=\left[\begin{array}{cc}1, & 0_{m-1}\end{array}\right]^{T}$. The state observer in the RSS form of (31) is designed as follows

$$
\begin{aligned}
\hat{p}(t)= & A_{m}^{T} \dot{p}(t)+\frac{1}{\bar{b}_{1}} \bar{B}_{m}\left(\dot{p}_{m}(t)-\bar{b}_{p}^{T} A_{m}^{T} \dot{p}(t)\right. \\
& -\bar{a}_{p}^{T} A_{n}^{T} \dot{x}(t)-\frac{a_{21}}{a_{11}} \dot{x}_{n}(t)+\frac{a_{21}}{a_{11}} \hat{\theta}^{T} \xi(t) \\
& -u(t))+c_{e_{p}}(\dot{p}(t)-\dot{\hat{p}}(t))
\end{aligned}
$$

where $c_{e_{p}}>0$ and in state space form it is written as

$$
\begin{aligned}
\dot{\hat{p}}(t)= & -\frac{1}{c_{e_{p}}} \hat{p}(t)+\frac{1}{c_{e_{p}}}\left(A_{m}^{T}+c_{e_{p}} I_{m}-\frac{1}{\bar{b}_{1}} \bar{B}_{m}\right. \\
& \left.\times \bar{b}_{p}^{T} A_{m}^{T}\right) \dot{p}(t)+\frac{1}{c_{e_{p}} \bar{b}_{1}} \bar{B}_{m}\left(\dot{p}_{m}(t)-\bar{a}_{p}^{T}\right. \\
& \left.\times A_{n}^{T} \dot{x}(t)-\frac{a_{21}}{a_{11}} \dot{x}_{n}(t)+\frac{a_{21}}{a_{11}} \hat{\theta}^{T} \xi(t)-u(t)\right) .
\end{aligned}
$$

The signals $\hat{p}(t)$ and $\hat{\theta}(t)$ are the estimates of unmeasured signal $p(t)$ and unknown constant $\theta$, respectively. Although the signal $\dot{p}(t)$ is measured, the signal $\dot{\hat{p}}(t)$ is also needed for representing the dynamics of the state observer and the observer error systems. Defining

$$
\begin{aligned}
e_{p}(t) & =p(t)-\hat{p}(t), \\
\widetilde{\theta}(t) & =\theta-\hat{\theta}(t),
\end{aligned}
$$

and subtracting (31) from (32), we obtain the error dynamics of the state observer in the RSS form as follows, 


$$
\begin{aligned}
e_{p}(t)= & -c_{e_{p}} \dot{e}_{p}(t)+\frac{a_{21}}{\bar{b}_{1} a_{11}} \bar{B}_{m}\left(\widetilde{\theta}^{T}(t) \xi(t)\right. \\
& \left.+\theta^{T} \delta(t)\right)
\end{aligned}
$$

The update law for $\hat{\theta}$ is designed as follows

$$
\dot{\hat{\theta}}(t)=\kappa \frac{a_{21}}{\bar{b}_{1} a_{11}} \xi(t) \bar{B}_{m}^{T} \dot{e}_{p}(t)
$$

where $\kappa>0$. It should be noted that update law, $\dot{\hat{\theta}}(t)$, is implementable since all signals given in (37) are measured including $\dot{e}_{p}(t)$.

Comparing the state observer given in [21] and given in (32), it is seen that they are not similar. Since the dimension of $p(t)$ is restricted as one in [21], the observer designed can not be used for the given problem in this note.

We state the following theorem for the designed observers.

Theorem 1. Consider the system dynamics (2), filter (21), (22), state observer (33) and the update law (37), under Assumptions 1-3, for initial conditions $p(0) \in \mathbb{R}^{m}, \hat{\theta}(0) \in \mathbb{R}^{2 q}, e_{p}(0) \in \mathbb{R}^{m}$, the signals $e_{p}(t), \widetilde{\theta}(t), \delta(t), \nu(t)-\hat{\theta}^{T}(t) \xi(t)$ converge to zero as $t \rightarrow \infty$.

Before proving the theorem, we state the following lemma since it is used in the proof of theorems.

Lemma 2. There exists $\rho>0$ such that for all $t_{0} \geq 0$, the following holds

$$
\begin{aligned}
Q_{p}\left(\rho, t_{0}\right)= & \int_{t_{0}}^{t_{0}+\rho} \xi(t) \xi^{T}(t) d t \\
& -\frac{1}{\rho} \int_{t_{0}}^{t_{0}+\rho} \xi(t) d t \int_{t_{0}}^{t_{0}+\rho} \xi^{T}(t) d t>0 .
\end{aligned}
$$

\section{Proof.}

$$
\dot{\xi}(t)=G \xi(t)+l \dot{\nu}(t),
$$

where $\dot{\nu}(t)=\sum_{i=1}^{q} \omega_{i} g_{i} \cos \left(\omega_{i} t+\phi_{i}\right)$. The proof for the signal given by (40) is given in 19. Using (26), time derivative of (25) yields

$$
\dot{\xi}(t)=G \xi(t)+l \dot{\nu}(t),
$$

where $\dot{\nu}(t)=\sum_{i=1}^{q} \omega_{i} g_{i} \cos \left(\omega_{i} t+\phi_{i}\right)$. The proof for the signal given by (40) is given in [19].
Proof of Theorem 1. We show the stability together with the convergence. The closed-loop system of $\left(e_{p}(t), \widetilde{\theta}(t)\right)$ is written as

$$
\dot{\zeta}(t)=E(t) \zeta(t)+F(t) \delta(t)
$$

where

$$
\begin{gathered}
E(t)=\left[\begin{array}{c}
-\frac{1}{c_{e_{p}}} I_{m} \\
\kappa \frac{a_{21}}{c_{e_{p}} \bar{b}_{1} a_{11}} \xi(t) \bar{B}_{m}^{T} \\
\frac{a_{21}}{c_{e_{p}} \bar{b}_{1} a_{11}} \bar{B}_{m} \xi(t)^{T} \\
-\frac{\kappa}{c_{e_{p}}}\left(\frac{a_{21}}{b_{1} a_{11}}\right)^{2} \xi(t) \xi(t)^{T}
\end{array}\right], \\
F(t)=\left[\begin{array}{c}
\frac{a_{21}}{c_{e_{p}} \bar{b}_{1} a_{11}} \bar{B}_{m} \theta^{T} \\
-\frac{\kappa}{c_{e_{p}}}\left(\frac{a_{21}}{b_{1} a_{11}}\right)^{2} \xi(t) \theta^{T}
\end{array}\right], \\
\zeta(t)=\left[\begin{array}{cc}
e_{p}(t), & \widetilde{\theta}^{T}(t)
\end{array}\right]^{T} .
\end{gathered}
$$

Since system matrix, $E(t)$ and input vector, $F(t)$ are time dependent, system (41) is a linear time varying system. The proof consists of two steps; first the homogeneous part is considered and the exponential stability of the equilibrium $\zeta(t)=0$ is shown, as the second step the signal convergence is proven by employing the results both given in Lemma 2 and the first step of the proof.

For the first step, we choose the Lyapunov function as follows

$$
V_{L T V}=\frac{1}{2} \zeta(t)^{T} P_{L T V} \zeta
$$

where

$$
P_{L T V}=\left[\begin{array}{cc}
I_{m} & 0_{m \times 2 q} \\
0_{2 q \times m} & \frac{1}{\kappa} I_{2 q}
\end{array}\right] .
$$

The time derivative of $V_{L T V}$ is given by

$$
\dot{V}_{L T V}=\zeta(t)^{T} C(t) C^{T}(t) \zeta(t) .
$$

where

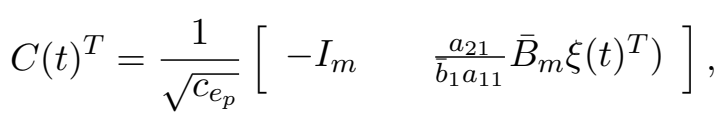

we get

$$
\begin{aligned}
\dot{V}_{L T V} & =\frac{1}{2} \zeta(t)^{T}\left(E^{T}(t) P_{L T V}+P_{L T V} E(t)\right) \zeta(t) \\
& =-\zeta(t)^{T} C(t) C^{T}(t) \zeta(t) \leq 0 .
\end{aligned}
$$

This proves the stability of equilibrium. However, we can not make any conclusion on the exponential stability. Therefore, we need to employ the 
series of theorems and lemmas given in 25$]$. Using (49), we obtain

$$
E^{T}(t) P_{L T V}+P_{L T V} E(t)+\alpha C^{T}(t) C(t) \leq 0
$$

for some $\alpha>0$. The exponential stability of the equilibrium of the homogeneous part of 41) is proven by showing the uniform complete observable (UCO) property of the pair $(C(t), E(t))$ [25]. In order to simplify the problem, we employ the following property which is given as follows; for a bounded $H(t)$, the pairs $(C(t), E(t))$ and $\left(C(t), E(t)+H(t) C(t)^{T}\right)$ have the same (UCO) property 25]. Choosing $H(t)=C(t)$, the system for the pair $\left(C(t), E(t)+H(t) C(t)^{T}\right)$ is written as

$$
\begin{aligned}
\dot{Y}(t) & =0, \\
y(t) & =C(t)^{T} Y(t)
\end{aligned}
$$

The state transition matrix of (51) is $\Phi(t)=$ $I_{(m+2 q)}$. Therefore, $\left(C(t), E(t)+H(t) C(t)^{T}\right)$ is a UCO pair if there exist $\alpha_{2}, \alpha_{3}, \rho>0$, such that the observability gramian satisfies

$$
\alpha_{2} I \geq \int_{t_{0}}^{t_{0}+\rho} C(t) C^{T}(t) d t \geq \alpha_{3} I,
$$

for all $t_{0} \geq 0$ [25]. The boundedness of $\xi(t)$ can be shown by (40) then, recalling (48), the upper bound of (53) is satisfied. We show the lower bound in (53). Calculating the integral in (53) yields

$$
\begin{aligned}
& X=\int_{t_{0}}^{t_{0}+\rho} C(t) C^{T}(t) d t \\
& =\frac{1}{c_{e_{p}}}\left[\begin{array}{c}
\rho I_{m} \\
-\frac{a_{21}}{b_{1} a_{11}} \int_{t_{0}}^{t_{0}+\rho} \xi(t) d t \bar{B}_{m}^{T}
\end{array}\right. \\
& \left.\begin{array}{c}
-\frac{a_{21}}{b_{1} a_{11}} \bar{B}_{m} \int_{t_{0}}^{t_{0}+\rho} \xi^{T}(t) d t \\
\left(\frac{a_{21}}{b_{1} a_{11}}\right)^{2} \int_{t_{0}}^{t_{0}+\rho} \xi(t) \xi^{T}(t) d t
\end{array}\right] .
\end{aligned}
$$

The lower bound argument is shown by concluding that $X$ is positive definite. To this end, we use Schur complement. The Schur complement of $\rho I_{m}$ of block matrix $X$ is given by,

$$
\begin{aligned}
S_{h}=\frac{1}{c_{e_{p}}}\left(\frac{a_{21}}{\bar{b}_{1} a_{11}}\right)^{2} & \left(\int_{t_{0}}^{t_{0}+\rho} \xi \xi^{T} d t\right. \\
& \left.-\frac{1}{\rho} \int_{t_{0}}^{t_{0}+\rho} \xi d t \int_{t_{0}}^{t_{0}+\rho} \xi^{T} d t\right) .
\end{aligned}
$$

According to Schur compliment, it is concluded that $X$ is positive definite if and only if $S_{h}$ is positive definite by denoting $I_{m} \rho$ is a positive definite matrix. We need to show that $S_{h}$ is positive definite. Noting that $\frac{1}{c_{e_{p}}}\left(\frac{a_{21}}{b_{1} a_{11}}\right)^{2}$ is a positive scalar, according to Lemma 2 there exists a positive $\rho$ such that for all $t_{0}>0$, then $S_{h}>0$. Hence, $\left(C, E+H C^{T}\right)$ is UCO, which implies that $(C, E)$ is UCO. Therefore, the state transition matrix $\Phi\left(t, t_{0}\right)$ corresponding to $E(t)$ in 41 satisfies

$$
\left\|\Phi\left(t, t_{0}\right)\right\| \leq \kappa_{0} e^{-\gamma_{0}\left(t-t_{0}\right)}
$$

for some positive constants $\kappa_{0}, \gamma_{0}$. Recalling that $G$ is Hurwitz, the solution of (26) yields

$$
|\delta(t)|=\left|e^{G\left(t-t_{0}\right)} \delta(0)\right| \leq \kappa_{1} e^{-\gamma_{1}\left(t-t_{0}\right)}|\delta(0)|
$$

for some positive constants $\kappa_{1}, \gamma_{1}$. Solving (41) yields

$$
\zeta(t)=\Phi(t, 0) \zeta(0)+\int_{0}^{t} \Phi(t, \tau) F(\tau) \delta(\tau) d \tau .
$$

Since $\xi(t)$ is bounded, from (40), it is concluded that $F(t)$ is bounded. Using (57)-(59), we get

$$
\begin{aligned}
|\zeta(t)| \leq & \kappa_{0} e^{-\gamma_{0} t}|\zeta(0)|+\frac{\kappa_{1} \kappa_{0} \sup _{0 \leq \tau \leq t}|F(\tau)|}{\left(\gamma_{0}-\frac{1}{2} \min \left\{\gamma_{0}, \gamma_{1}\right\}\right)}|\delta(0)| \\
& \times e^{-\frac{1}{2} \min \left\{\gamma_{0}, \gamma_{1}\right\} t}
\end{aligned}
$$

Finally, by considering 60 , we prove that $\zeta(t)=$ $\left[e_{p}(t), \quad \widetilde{\theta}^{T}(t)\right]^{T}$ converge to zero as $t \rightarrow \infty$. Since $\widetilde{\theta}(t)$ tends to zero, in other words, the perfect estimation of unknown constant parameter $\theta$ is achieved, from Lemma 1, and noting that $\delta(t)$ converges to zero, we also prove that $\nu(t)-\hat{\theta}^{T}(t) \xi(t)$.

The result of Theorem 1 enables us to estimate unknown disturbance signal $\nu(t)$ with unmeasured state $p(t)$ for an open loop case. The perfect estimation relies on the excitation of all modes of the disturbance signal. This provides the persistence of excitation that is given in Lemma 2.

In the next section, the algorithm for the input $u(t)$ to cancel the disturbance in the system is given.

\section{Active disturbance cancellation}

We design an active disturbance cancellation algorithm for the actual input $u(t)$ by employing a backstepping technique. This technique is firstly 
proposed by Petar Kokotovic for the control design of nonlinear system which are in strict feedback form [26]. However, the usage of this method is not restricted by only a special class of nonlinear systems. In [27], adaptive controller design methodology without any over parametrization for systems that contain parametric uncertainties which are unmatched with the actual input is given based on aforementioned technique. The idea behind the backstepping is to find a control law that transform the closed loop system to a desired form step by step, this can also be seen as recursive method. Particularly, in the considered problem, firstly we design a law for the virtual control input $p(t)$ then by backstepping, we reach the actual control input. It is called virtual control, since it is not the actual input but it is seen as the input of the subsystem. Therefore, the main role of actual input to drive the virtual input so that the state of the considered system is driven to a desired set. Since $p(t)$, is not measured, this technique can not be applied directly. This problem is handled by using the result obtained in the previous section. Using (36) and (34), we represent the unmeasured $p(t)$ as follows,

$$
\begin{aligned}
p(t)= & \hat{p}(t)-c_{e_{p}} \dot{e}_{p}(t)+\frac{a_{21}}{\bar{b}_{1} a_{11}} \bar{B}_{m}\left(\widetilde{\theta}^{T}(t) \xi(t)\right. \\
& \left.+\theta^{T} \delta(t)\right)
\end{aligned}
$$

\subsection{Backstepping design}

We can now consider the problem as an adaptive control design. Substituting (19), (61) into 27) and recalling $\theta=\widetilde{\theta}(t)+\hat{\theta}(t)$ from 35 , we obtain

$$
\begin{aligned}
x(t)= & A_{n}^{T} \dot{x}(t)+\frac{1}{a_{11}} \bar{B}_{n}\left(-b_{x}^{T} \hat{p}(t)+c_{e_{p}} b_{x}^{T} \dot{e}_{p}(t)\right. \\
& +\dot{x}_{n}(t)-a_{x}^{T} A_{n}^{T} \dot{x}(t)-\hat{\theta}^{T} \xi(t) \\
& \left.-\left(\frac{a_{21} b_{11}}{\bar{b}_{1} a_{11}}+1\right)\left(\widetilde{\theta}^{T} \xi(t)+\theta^{T} \delta(t)\right)\right) .
\end{aligned}
$$

It is possible to consider $b_{x}^{T} \hat{p}(t)$ as the virtual controller for a backstepping design since the dynamic of $\hat{p}(t)$ depends on only measured signals.

The backstepping procedure consist of the following steps; (1) designing a controller $b_{x}^{T} \hat{p}(t)$ that both cancels the disturbance and stabilize the system, (2) defining the error between the desired and actual value $b_{x}^{T} \hat{p}(t)$, this step can be considered as a state transformation, (3) by taking the time derivative of the defined error term, we find the dynamics of error. In the last step, we reach the actual control input.

First Step: The desired value of $b_{x}^{T} \hat{p}(t)$ is designed by

$\hat{p}_{d}(t)=-a_{11} K \dot{x}(t)-\hat{\theta}^{T}(t) \xi(t)+\dot{x}_{n}(t)-a_{x}^{T} A_{n}^{T} \dot{x}(t)$,

where the control gain $K=\left[k_{1}, \cdots, k_{n}\right] \in \mathbb{R}^{1 \times n}$ is chosen so that $\left(A_{n}^{T}+\bar{B}_{n} K\right)$ is Hurwitz with $P=P^{T}>0$ that is the solution of

$$
A_{c l}{ }^{-T} P+P A_{c l}{ }^{-1}=-2\left(2+\frac{c_{e_{p}}}{2}\right) I .
$$

where

$$
A_{c l}=\left(A_{n}^{T}+\bar{B}_{n} K\right)^{-1} \text {. }
$$

Second Step: we define the following error term,

$$
e_{d}(t)=b_{x}^{T} \hat{p}(t)-\hat{p}_{d}(t) .
$$

Third Step: Taking the derivative of (66), we obtain

$$
\begin{aligned}
\dot{e}_{d}(t)= & -\frac{1}{c_{e_{p}}} b_{x}^{T} \hat{p}(t)+\left(\frac { 1 } { c _ { e _ { p } } } b _ { x } ^ { T } \left(A_{m}^{T}+c_{e_{p}} I_{m}\right.\right. \\
& \left.\left.-\frac{1}{\bar{b}_{1}} \bar{B}_{m} \bar{b}_{p}^{T} A_{m}^{T}\right)+\left(a_{11} k_{n}-1\right) b_{x}^{T}\right) \dot{p}(t) \\
& +\left(\frac{-b_{11}}{c_{e_{p}} \bar{b}_{1}} \bar{a}_{p}^{T} A_{n}^{T}+a_{11} K A_{n}\right. \\
& \left.+a_{x}^{T} A_{n}^{T} A_{n}+\left(a_{11} k_{n}-1\right) a_{x}^{T}\right) \dot{x}(t) \\
& +\frac{b_{11} a_{21}}{c_{e_{p}} \bar{b}_{1} a_{11}} \hat{\theta}^{T}(t) \xi(t)+\hat{\theta}^{T}(t) G \xi(t) \\
& +\dot{\hat{\theta}}^{T}(t) \xi(t)+\left(\hat{\theta}^{T}(t) l+\left(a_{11} k_{n}-1\right)\right) \\
& \times\left(\beta^{T} \xi(t)+\beta^{T} \delta(t)\right)+\frac{b_{11}}{c_{e_{p}} \bar{b}_{1}}\left(\dot{p}_{m}(t)\right. \\
& \left.-\frac{a_{21}}{a_{11}} \dot{x}_{n}(t)-u(t)\right) .
\end{aligned}
$$

The representation (20) is used for $\dot{\nu}(t)$. In this way, we transform $(x(t), p(t))$ system into $\left(x(t), e_{p}(t), e_{d}(t)\right)$ system where the uncertainties are now matched with the real actuator.

As it is realized from (66), if $e_{d}(t)$ converges to zero, the virtual controller tends to its desired value which cancels the disturbance effect and stabilize system (1).

In the next section, the adaptive controller and the main theorem are given. 


\subsection{Adaptive controller}

Considering the transformed system (1), (36) and (67) and their RSS forms, we design the following adaptive controller

$$
\begin{aligned}
u= & \dot{p}_{m}(t)-\frac{a_{21}}{a_{11}} \dot{x}_{n}(t)+\frac{c_{e_{p}} \bar{b}_{1}}{b_{11}}\left(-\frac{1}{c_{e_{p}}} b_{x}^{T} \hat{p}(t)\right. \\
& +\left(\frac{1}{c_{e_{p}}} b_{x}^{T}\left(A_{m}^{T}+c_{e_{p}} I_{m}-\frac{1}{\bar{b}_{1}} \bar{B}_{m} \bar{b}_{p}^{T} A_{m}^{T}\right)\right. \\
& \left.+\left(a_{11} k_{n}-1\right) b_{x}^{T}\right) \dot{p}(t)+\left(\frac{-b_{11}}{c_{e_{p}} \bar{b}_{1}} \bar{a}_{p}^{T} A_{n}^{T}\right. \\
& \left.+a_{11} K A_{n}+a_{x}^{T} A_{n}^{T} A_{n}+\left(a_{11} k_{n}-1\right) a_{x}^{T}\right) \dot{x}(t) \\
& +\frac{b_{11} a_{21}}{c_{e_{p}} \bar{b}_{1} a_{11}} \hat{\theta}^{T}(t) \xi(t)+\hat{\theta}^{T}(t) G \xi(t)+\dot{\hat{\theta}}^{T}(t) \xi(t) \\
& +\left(\hat{\theta}^{T}(t) l+\left(a_{11} k_{n}-1\right)\right) \hat{\beta}^{T}(t) \xi(t) \\
& -\frac{1}{a_{11}} \dot{x}^{T}(t) P \bar{B}_{n} \\
& \left.+\left(\frac{1}{2}\left(\hat{\theta}^{T}(t) l+\left(a_{11} k_{n}-1\right)\right)^{2}+c_{e_{d}}\right) e_{d}(t)\right)
\end{aligned}
$$

where $c_{e_{d}}>0$. The dynamics to update parameter estimation is given by

$$
\begin{aligned}
\dot{\hat{\theta}}= & \kappa_{\theta} \frac{1}{\bar{b}_{1} a_{11}} \xi(t)\left(a_{21} \bar{B}_{m}^{T} \dot{e}_{p}(t)\right. \\
& \left.-\frac{a_{21} b_{11}-\bar{b}_{1} a_{11}}{\gamma a_{11}} \dot{x}(t)^{T} P \bar{B}_{n}\right), \\
\dot{\hat{\beta}}= & \kappa_{\beta}\left(\hat{\theta}^{T}(t) l+\left(a_{11} k_{n}-1\right)\right) \xi(t) e_{d}(t) .
\end{aligned}
$$

with

$$
\gamma=\frac{1}{a_{11}^{2}} \lambda_{\max }\left(\bar{B}_{n}^{T} P b_{x}^{T} b_{x} P \bar{B}_{n}\right) .
$$

Both control and update laws consist of only measured signals.

As it is seen by comparing the controller $(68)$ and the one designed in 21], they are different than each other. It is not possible to employ the controller in 21] to stabilize the system given in the problem statement of this note.

Remark 1. Although parameter $\theta$ represents the same property, the update laws given for $\hat{\theta}(t)$ in the open loop estimation and the closed loop cancellation are slightly different. The update law (69) employed in closed loop cancellation contains an extra term, which is given by $-\kappa_{\theta} \xi(t) \frac{a_{21} b_{11}-\bar{b}_{1} a_{11}}{\bar{b}_{1} a_{11}^{2}} \dot{x}(t)^{T} P \bar{B}_{n}$. The backstepping technique causes the extra term in the update law.
Remark 2. We design the control and update laws for the case where all state-derivative measurements are accurate. That might not be the case in the implementation of algorithms to actual systems, since the measurements providing by sensors in the applications may contain noise unless an appropriate filtering is applied. In adaptive control, measurement noise and unmodeled dynamics may harm the stability of the equilibrium due to drift in the estimation of the parameters. However, there exist simple robustification tools for mostly update laws to eliminate the effect of noise and unmodeled dynamics [25]. One of the appropriate robustification tools can be employed together with the proposed algorithm to provide robustness with respect to measurement noise and unmodeled dynamics. The main drawback of the these tools is the trade off between robustness and the convergence.

We define the following signal

$$
\widetilde{\xi}=\xi-\bar{\xi}
$$

where $\bar{\xi}=\int_{0}^{t} e^{G(t-\tau)} G l \dot{\nu}(\tau) d \tau$ that is used in the stability statement.

Theorem 2. Consider closed-loop system that is composed of the plant (1), (2) driven by the unknown disturbance signal (8), (9), the disturbance observer dynamics (21), (22), the state observer (33) and the adaptive controller (68)-(70). Under Assumptions 1 and 2, the followings hold:

- For all initial conditions, all signals are bounded and $\dot{x}(t), \dot{e}_{p}(t), e_{d}(t), \widetilde{\xi}, \delta$ converge to zero as $t \rightarrow \infty$,

- In addition, for all $w(0) \in \mathbb{R}^{2 q}$ such that Assumption 3 holds, the signals $x(t), e_{p}(t), \widetilde{\theta}, \nu(t)-\hat{\theta}^{T} \xi$ converge to zero as $t \rightarrow \infty$.

\section{Stability proof}

The proof of Theorem 2 is given as follows.

Proof of Theorem 2: The transformed closed system with the state observer is given as follows

$$
\begin{aligned}
\dot{x}(t)= & A_{c l} x(t)-\frac{1}{a_{11}} A_{c l} \bar{B}_{n}\left(-e_{d}(t)+c_{e_{p}} b_{x}^{T} \dot{e}_{p}(t)\right. \\
& \left.-\left(\frac{a_{21} b_{11}}{\bar{b}_{1} a_{11}}+1\right)\left(\widetilde{\theta}^{T} \xi(t)+\theta^{T} \delta(t)\right)\right),
\end{aligned}
$$




$$
\begin{aligned}
\dot{e}_{p}(t)= & -\frac{1}{c_{e_{p}}} e_{p}(t)+\frac{a_{21}}{c_{e_{p}} \bar{b}_{1} a_{11}} \bar{B}_{m}\left(\widetilde{\theta}^{T}(t) \xi(t)\right. \\
& \left.+\theta^{T} \delta(t)\right), \\
\dot{e}_{d}= & -\left(\frac{1}{2}\left(\hat{\theta}^{T}(t) l+\left(a_{11} k_{n}-1\right)\right)^{2}+c_{e_{d}}\right) e_{d}(t) \\
& +\left(\hat{\theta}^{T}(t) l+\left(a_{11} k_{n}-1\right)\right)\left(\widetilde{\beta}^{T}(t) \xi(t)\right. \\
& \left.+\beta^{T} \delta(t)\right)+\frac{1}{a_{11}} \dot{x}^{T}(t) P \bar{B}_{n}
\end{aligned}
$$

where

$$
\widetilde{\beta}(t)=\hat{\beta}(t)-\beta .
$$

We consider the following Lyapunov function,

$$
\begin{aligned}
V= & \frac{1}{2}\left(x^{T} P x+\gamma e_{p}^{2}+e_{d}^{2}+\frac{\gamma}{\kappa_{\theta}} \widetilde{\theta}^{T} \widetilde{\theta}+\frac{1}{\kappa_{\beta}} \widetilde{\beta}^{T} \widetilde{\beta}\right. \\
& \left.+\epsilon_{\delta} \delta^{T} P_{\delta} \delta\right)
\end{aligned}
$$

where

$$
\begin{aligned}
G^{T} P_{\delta}+ & P_{\delta} G=-2 I \\
\epsilon_{\delta}= & \left(\frac{a_{21} b_{11}}{\bar{b}_{1} a_{11}^{2}}+\frac{1}{a_{11}}\right)^{2} \lambda_{\max }\left(\theta \bar{B}_{n}^{T} P P \bar{B}_{n} \theta^{T}\right) \\
& +\left(\gamma \frac{a_{21} b_{11}}{\bar{b}_{1} a_{11}}\right)^{2} \lambda_{\max }\left(\theta \bar{B}_{m} \bar{B}_{m}^{T} \theta^{T}\right) \\
& +\lambda_{\max }\left(\beta \beta^{T}\right) .
\end{aligned}
$$

The derivative of $V$ with respect to time, in view of $(20),(69), 770)$ and $(73)-(75)$, is given by

$$
\begin{aligned}
\dot{V}= & -\left(2+\frac{c_{e_{p}}}{2}\right) \dot{x}^{T}(t) \dot{x}(t)-\gamma c_{e_{p}} \dot{e}_{p}^{T}(t) \dot{e}_{p}(t) \\
& -\left(\frac{1}{2}\left(\hat{\theta}^{T}(t) l+\left(a_{11} k_{n}-1\right)\right)^{2}+c_{e_{d}}\right) e_{d}^{2}(t) \\
& +\frac{1}{a_{11}} \dot{x}^{T} P \bar{B}_{n}\left(c_{e_{p}} b_{x}^{T} \dot{e}_{p}(t)-\left(\frac{a_{21} b_{11}}{\bar{b}_{1} a_{11}}+1\right) \theta^{T} \delta(t)\right. \\
& +\frac{a_{21}}{\bar{b}_{1} a_{11}} \dot{e}_{p}^{T} \bar{B}_{m} \theta^{T} \delta+\left(\hat{\theta}^{T}(t) l+\left(a_{11} k_{n}-1\right)\right) \\
& \times\left(\widetilde{\beta}^{T}(t) \xi(t)+\beta^{T} \delta(t)\right) \beta^{T} \delta e_{d}-\epsilon_{\delta}^{2} \delta^{T} \delta
\end{aligned}
$$

Using Young's inequality, we get

$$
\begin{aligned}
\dot{V} \leq & -\dot{x}^{T}(t) \dot{x}(t)-\frac{\gamma c_{e_{p}}}{2} \dot{e}_{p}^{T}(t) \dot{e}_{p}(t)-c_{e_{d}} \\
& e_{d}^{2}(t)-\frac{\epsilon_{\delta}}{2} \delta^{T}(t) \delta(t) .
\end{aligned}
$$

From 81), we conclude

$$
V(t) \leq V(0)
$$

Defining

$$
\Theta(t)=\left[x^{T}(t), e_{p}(t), e_{d}(t), \widetilde{\theta}^{T}(t), \widetilde{\beta}^{T}(t), \delta^{T}(t)\right]^{T},
$$

and using (77) and 82 , we get

$$
|\Theta(t)|^{2} \leq M_{1}|\Theta(0)|^{2},
$$

for some $M_{1}>0$. Taking derivative of $(72)$ and using 40) yield

$$
\dot{\widetilde{\xi}}(t)=G \widetilde{\xi}(t)
$$

Since $G$ is Hurwitz, using (85), we get

$$
|\widetilde{\xi}(t)| \leq M_{2} \mathrm{e}^{-\alpha_{1} t}|\widetilde{\xi}(0)|,
$$

for some $M_{2}, \alpha_{1}>0$. By using (84) and (86), we obtain

$$
|\Xi(t)| \leq M_{4}|\Xi(0)|
$$

where

$$
\Xi(t)=\left[\Theta^{T}(t), \widetilde{\xi}^{T}(t)\right]^{T},
$$

for some $M_{4}>0$. From (87), it is concluded that all signals are bounded for all initial conditions. Since the closed loop dynamics given by 73 - 75 are continuous in $\Xi$ and $t,(81)$ is also continuous in $\Xi$ and $t$. Moreover, 81 is zero at $\Xi=0$. Therefore, we conclude that $\dot{x}(t), \dot{e}_{p}(t), e_{d}(t)$ and T) converge to zero as $t \rightarrow \infty$, by the LaSalleYoshizawa theorem. This proves part 2 of Theorem 2.

We now prove the convergence of $x(t)$. To this end we first need to show the convergence of $\left.e_{p}(t), \widetilde{\theta}(t)\right)$. This part is similar to the proof of Theorem 1. The system of $\left(e_{p}(t), \widetilde{\theta}(t)\right)$ is written as

$$
\dot{\zeta}(t)=E(t) \zeta(t)+F_{d}(t) d(t),
$$

where $E(t)$ and $\zeta(t)$ are given by (42) and (44), respectively and 


$$
\begin{gathered}
F_{d}(t)=\left[\begin{array}{c}
0_{m \times n} \\
\kappa_{\theta} \frac{a_{2} 1 b_{1} 1-\bar{b}_{1} a_{1} 1}{\gamma a_{1}^{2} 1 \bar{b}_{1}} \xi(t) \bar{B}_{n}^{T} P \\
\frac{a_{21}}{c_{e_{p}} \bar{b}_{1} a_{11}} \bar{B}_{m} \theta^{T} \\
-\frac{\kappa_{\theta}}{c_{e_{p}}}\left(\frac{a_{21}}{\bar{b}_{1} a_{11}}\right)^{2} \xi(t) \theta^{T}
\end{array}\right], \\
d(t)=\left[\begin{array}{ll}
\dot{x}(t), & \delta^{T}(t)
\end{array}\right]^{T} .
\end{gathered}
$$

As it is seen the the homogeneous part of systems (41) and (89) are same. The only difference is the input matrices. We have already shown the exponential stability of the homogeneous part in the proof of Theorem 1. The boundedness of $d(t)$ and $F_{d}(t)$ is concluded from (91) and $(90)$, respectively. Noting that $d(t)$ goes to zero, from (89) and (57), it follows that $\zeta(t)$ is bounded and $\zeta(t)=\left[\begin{array}{cc}e_{p}(t), & \widetilde{\theta}^{T}(t)\end{array}\right]^{T} \rightarrow 0$ as $t \rightarrow \infty$. Since $\delta(t), \widetilde{\theta}(t), \dot{e}_{p}(t), e_{d}(t) \rightarrow 0$ and $A_{c l}$ is Hurwitz, from (73), it is shown that $x(t)$ converges to zero as $t \rightarrow \infty$. In addition, from (19), we conclude that $\hat{\theta}^{T}(t) \xi(t)-\nu(t) \rightarrow 0$ as $t \rightarrow \infty$. This proves part 2 of Theorem 2 .

\section{Example}

We consider a two-degree of freedom system which is illustrated in Figure 1, as a simulation example.

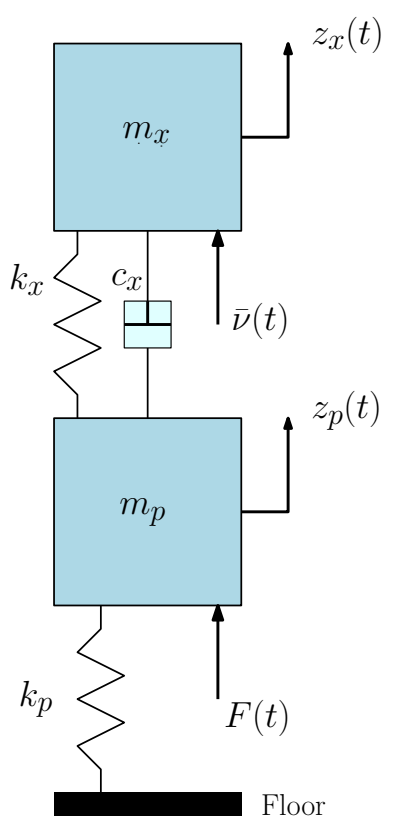

Figure 1. The dynamical system considered for numerical example.
The mass denoted by $m_{x}$ is forced by an unknown disturbance $\bar{\nu}(t)$ and connected to another mass, $m_{p}$ which is actuated by a force input. The system dynamics are given by

$$
\begin{aligned}
m_{x} \ddot{z}_{x}(t)= & -k_{x}\left(z_{x}(t)-z_{p}(t)\right) \\
& -c_{x}\left(\dot{z}_{x}(t)-\dot{z}_{p}(t)\right)+\nu(t), \\
m_{p} \ddot{z}_{p}(t)= & -k_{p} z_{p}+k_{x}\left(z_{x}(t)-z_{p}(t)\right) \\
& +c_{x}\left(\dot{z}_{x}(t)-\dot{z}_{p}(t)\right)+F(t) .
\end{aligned}
$$

Choosing the state as $x(t)=\left[\begin{array}{ll}z_{x}(t), & \dot{z}_{x}(t)\end{array}\right]^{T}$ and $p(t)=\left[\begin{array}{ll}z_{p}(t), & \dot{z}_{p}(t)\end{array}\right]^{T}$, we obtain $a_{x}^{T}=$ $\left[-\frac{k_{x}}{m_{x}},-\frac{c_{x}}{m_{x}}\right], \quad b_{x}^{T}=\left[\frac{k_{x}}{m_{x}}, \frac{c_{x}}{m_{x}}\right], a_{p}^{T}=$

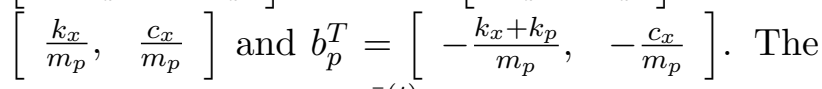
disturbance is $\nu(t)=\frac{\bar{\nu}(t)}{m_{x}}$ and the input is $u(t)=$ $\frac{F(t)}{m_{p}}$. The system parameters are chosen as $m_{x}=$ $10, m_{p}=5, k_{x}=10, k_{p}=5, c_{x}=10$. The mass denoted by $m_{x}$ is driven by the unknown periodic disturbance $\bar{\nu}(t)$ and the states of mass $m_{p}$ through the connected spring and damper. The mass $m_{x}$ is the subject system to stabilize. The effect of the input $F(t)$ reaches the main system through the dynamics of $m_{p}$ whose order is more than one. Therefore, it should be noted that the design given in [21] can not be used for this example. By employing the developed algorithms, we present the results for estimation and active disturbance cancellation.

\subsection{Observer based disturbance and state estimation}

In this case, the only aim is estimation. The unknown disturbance signal $\nu(t)=\frac{\bar{\nu}(t)}{m_{x}}=$ $\sin \left(\frac{2 \pi}{3.5} t\right)+0.5 \sin \left(\frac{2 \pi}{4} t+\frac{\pi}{4}\right)+0.5 \sin \left(\frac{2 \pi}{3} t+\pi\right)$ and initial condition $x(0)=p(0)=\left[\begin{array}{ll}-0.5 & -0.5\end{array}\right]^{T}$. The update and estimations gains is chosen as $\kappa=15000$ and $c_{e_{p}}=20$. The pair $(G, l)$ is $l=\left[0_{5}^{T}, 1\right]^{T}, G=\left[\begin{array}{cc}0_{5} & I_{5} \\ 0 & 0_{5}^{T}\end{array}\right]+$ $l[-527.8-1146.1-1024.2-482.5-126.4-17.5]^{T}$ which is controllable. Since the critical signal is the position of $m_{p}$, we have plotted its estimate in Figure 2. The estimation of the disturbance together with the actual signal are plotted in Figure 3, From Figures 2 and 3, it is seen that $\left|z_{p}(t)-\hat{z}_{p}(t)\right|$ converge to zero and the estimate of unknown disturbance, $\hat{\theta}^{T}(t) \xi(t)$ converges to the actual disturbance signal, thus perfect estimation is achieved, as Theorem 1 states. 


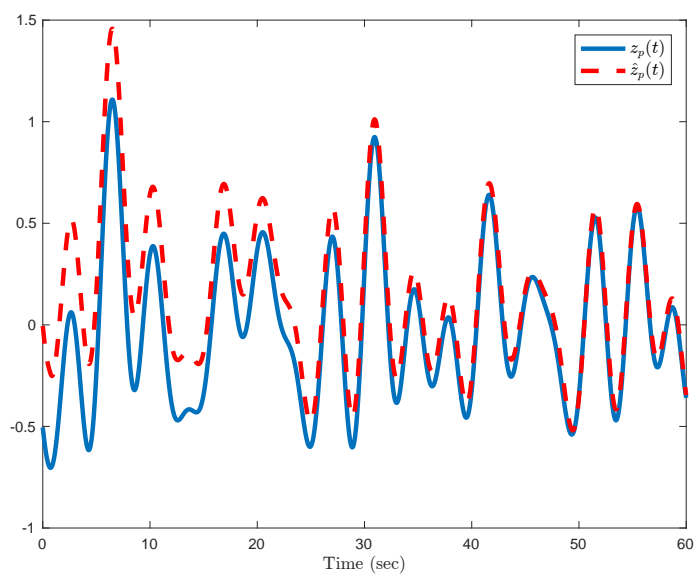

Figure 2. Estimation of the actuator state $\hat{z}_{p}(t)$ for the observer based estimation.

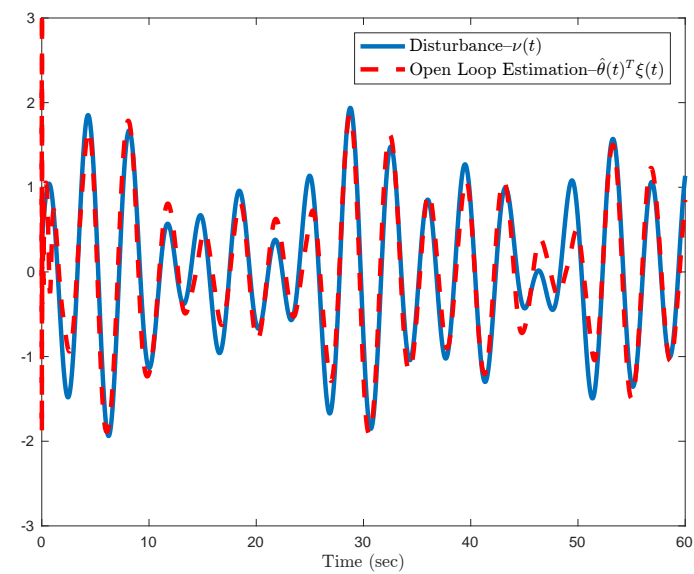

Figure 3. The actual disturbance signal $\nu(t)$ and its estimate, $\hat{\theta}^{T}(t) \xi(t)$, for the observer based estimation.

\subsection{Active cancellation}

In this case, the aim is to actuate mass $m_{p}$ by $F(t)$ so that the $z_{p}(t), \dot{z}(p)$ cancel the disturbance on mass $m_{x}$. The unknown disturbance $\nu(t)=$ $\frac{\bar{\nu}(t)}{m_{x}}=2 \sin \left(\frac{2 \pi}{3} t\right)+\sin \left(\frac{2 \pi}{t}+\frac{\pi}{4}\right)$ and the initial conditions of the states are chosen same as the first case. The control gain $K$ is chosen such that the eigenvalues of $A_{c l}^{-1}$ are -3 and -4 . The gains are $c_{e_{d}}=350, c_{e_{p}}=0.2, \kappa_{\theta}=50$ and $\kappa_{\beta}=10$. The pair $(G, l)$ is $l=\left[\begin{array}{ll}0_{3}^{T}, & 1\end{array}\right]^{T}, G=\left[\begin{array}{cc}0_{3} & I_{3} \\ 0 & 0_{3}^{T}\end{array}\right]+$ $l[-48.51-75.91-43.63-10.90]^{T}$ which is controllable. The state of mass $m_{x}$ is given in Figure 4. The result of the disturbance estimation is presented in Figure 5. From Figures 4 and 5 it is observed that $z_{x}(t), \dot{z}_{x}(t)$ converge to zero and the estimate of unknown disturbance, $\hat{\theta}^{T}(t) \xi(t)$ converges to the actual disturbance signal, thus perfect estimation is achieved, as Theorem 2 states.



Figure 4. Position, $z_{x}(t)$, and velocity, $\dot{z}_{x}(t)$, of mass $m_{x}$ when the cancellation algorithm is on.

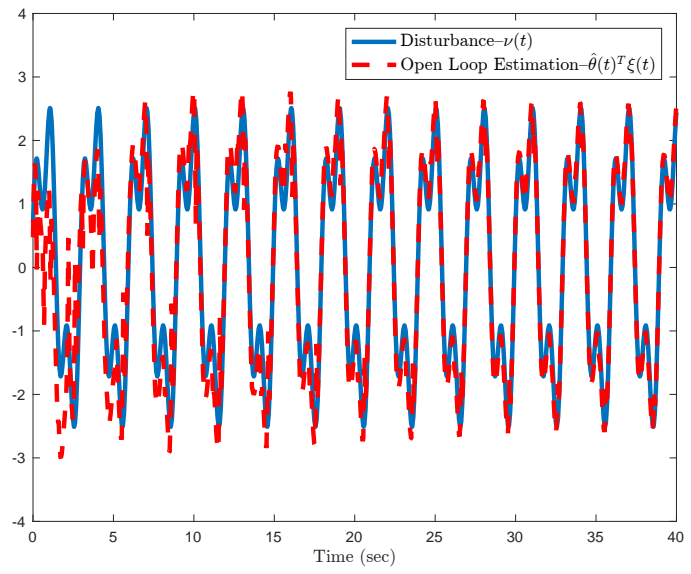

Figure 5. The actual disturbance signal $\nu(t)$ and its estimate, $\hat{\theta}^{T}(t) \xi(t)$, for the active cancellation. 


\section{Conclusions}

In this note, we consider an active disturbance cancellation problem for the case where the disturbance signal which is modeled as the sum of $q$ sinusoidal functions, and the real control input of the system are unmatched. Moreover, the only available measurements for the feedback are the state derivatives. Two algorithms are developed; the first one is to estimate the unmeasured disturbance and states in the open loop, the second one is to cancel the effect of the disturbance and maintain the state convergence in the closed loop case. To this end, observers are designed for both the unknown disturbance and the unmeasured state that appears as the virtual control input in the system. We perform a backstepping procedure to handle the unmatched condition and obtain the control and update laws by using the estimate of the unmeasured state as the virtual control input. The stability of the equilibrium of the closed-loop adaptive system and the the convergence of state derivatives, $\dot{x}$, to zero in time are shown. By assuming all modes of the disturbance signal are excited, we also achieve to prove that the state $x(t)$ converges to zero as $t \rightarrow \infty$ and the estimate of disturbance, $\hat{\theta}^{T}(t) \xi(t)$ converges to actual disturbance signal, $\nu(t)$. We illustrate the effectiveness of developed algorithms by performing numerical simulations for a two-degree of freedom springdamper-mass system. The simulation results also verify that the developed algorithms are working correctly as the given theorems state.

\section{References}

[1] Arslan Y.Z., Sezgin A.,\& Yagiz N.(2015). Improving the ride comfort of vehicle passenger using fuzzy sliding mode controller. Journal of Vibration and Control, 21(9), 1667-1679.

[2] Marconi, L., Isidori A., \& Serrani, A. (2002). Autonomous vertical landing on an oscillating platform: an internal-model based approach. Automatica, 38(1), 21-32.

[3] Basturk, H. I., \& Krstic,M. (2013). Adaptive wave cancellation by acceleration feedback for rampconnected air cushion-actuated surface effect ships. Automatica, 49(9), 2591-2602.

[4] Basturk, H. I., Rosenthal, B., \& Krstic, M. (2103). Pitch control design for tandem lifting body catamaran by aft lifting body actuation. IEEE Transactions on Control Systems Technology, 23(2), 700-707.

[5] Johnson, C. D. (1971). Accommodation of external disturbances in linear regulator and servomechanism problems. IEEE Transactions on Automatic Control, 16(6), pp. 635-644.

[6] Francis, D. A., \& Wonham, W. N. (1975). The internal model principle for linear multivariable regulators. Applied Mathematics and Optimization, 2(2), 170-194.
[7] Dogruel, M., \& Celik, H. H. (2011). Harmonic control arrays method with a real time application to periodic position control. IEEE Transactions on Control Systems Technology, 19(3), 521-530.

[8] Kim, H., \& Shim, H. (2015) "Linear systems with hyperbolic zero dynamics admit output regulator rejecting unknown number of unknown sinusoids," IET Control Theory and Applications, 9 , pp. 1472 - 1480, 2015.

[9] Marino, R., \& Santosuosso, G. L. (2007). Regulation of linear systems with unknown exosystems of uncertain model. IEEE Transactions on Automatic Control, 52(2), 353-359.

[10] Bodson. M., \& Douglas, S. (1997) Adaptive algorithms for the rejection of sinusoidal disturbances with unknown frequency. Automatica, 33(12), 2213-2221.

[11] Bobtsov A. A., \& Pyrkin, A. A. (2009). Compensation of unknown sinusoidal disturbances in linear plants of arbitrary relative degree. Automation and Remote Control, 70(3), 449-456.

[12] Marino, R., \& Tomei, P. (2005). Adaptive tracking and disturbance rejection for uncertain nonlinear systems. IEEE Transactions on Automatic Control, 50(1), 90-95.

[13] Marino, R., \& Santosuosso, G. L. (2005). Global compensation of unknown sinusoidal disturbances for a class of nonlinear nonminimum phase systems. IEEE Transactions on Automatic Control, 50(11), 18161822 .

[14] Nikiforov, V. O. (2001). Nonlinear servocompensation of unknown external disturbances. Automatica, $37(10), 1617-1653$.

[15] Ding, Z. (2003). Universal disturbance rejection for nonlinear systems in output feedback form. IEEE Transactions on Automatic Control, 48(7), 1222-1227.

[16] Kwak, S. K., Washington G., \& Yedavalli, R. K. (2002) Acceleration-based vibration control of distributed parameter systems using the 'reciprocal state-space framework. Journal od Sound and Vibration, 251(3), 543-557.

[17] Abdelaziz, T. H. S. (2008). Robust pole assignment for linear time-invariant systems using state-derivative feedback. Journal of Systems and Control Engineering, 223(2), 187-199.

[18] Michiels, W., Vyhlídal, T., Huijberts, H., \& Nijmeijer, H. (2009) Stabilizability and stability robustness of state derivative feedback controllers. SIAM Journal on Control and Optimization, 47(6), 3100-3117.

[19] Basturk, H. I., \& Krstic, M. (2013). Adaptive cancelation of matched unknown sinusoidal disturbances for LTI systems by state derivative feedback. Journal of Dynamic Systems, Measurement, and Control, 135(1), 014501-014507.

[20] Basturk, H. I., \& Krstic, M. (2012) Adaptive backstepping cancelation of unmatched unknown sinusoidal disturbances for LTI systems by state derivative feedback. ASME Dynamic Systems and Control Conference, 1-9.

[21] Basturk, H. I. (2015). Observer based adaptive estimation/cancellation of unmatched sinusoidal disturbances in known LTI systems by state derivative measurement. ASME Dynamic Systems and Control Conference, 2015. 
[22] Nikiforov, V. O. (2004). Observers of external deterministic disturbances. I. objects with known parameters. Automation and Remote Control, 65(10), 15311541.

[23] Chen, C. T. (1984) Linear System Theory and Design. Rinehart, Winston. New York, NY: Holt.

[24] Salem, A., \& Said, K. (2008). A simple proof of Sylvester's (Determinants) Identity. Applied Mathematical Sciences, 32(2), 1571-1580.

[25] Ioannou, P., \& Sun, J. (1996) Robust Adaptive Control. Prentice-Hall.

[26] Kokotovic, P.V. (1990). The joy of feedback: nonlinear and adaptive. IEEE Control Systems Magazine, 12(3): 717 .
[27] Krstic M., Kanellakopoulos I., \& Kokotovic, P. (1995) Nonlinear and Adaptive Control Design, Wiley.

Halil Ibrahim Basturk received his B.S. and M.S. degrees in Mechanical Engineering from Bogazici University in 2006 and 2008, and the Ph.D. degree in Mechanical and Aerospace Engineering from the University of California at San Diego in 2013. Since 2014 he has been an Assistant Professor of Mechanical Engineering at Bogazici University, Istanbul, Turkey. His research interests include disturbance estimation/cancellation, adaptive control, boundary control of infinite dimensional systems and extremum seeking.

An International Journal of Optimization and Control: Theories \& Applications (http://ijocta.balikesir.edu.tr)

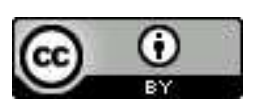

This work is licensed under a Creative Commons Attribution 4.0 International License. The authors retain ownership of the copyright for their article, but they allow anyone to download, reuse, reprint, modify, distribute, and/or copy articles in IJOCTA, so long as the original authors and source are credited. To see the complete license contents, please visit http://creativecommons.org/licenses/by/4.0/. 